actual units in any case will be determined by the unit that needs to be adopted in the case of the longest scale, to bring that scale within the limits of the page.

\title{
Summary.
}

Tabulated data may be interpolated graphically by means of logarithmic scales printed in the margins and in the middle of each page of the table. The space occupied by most tables may thus be reduced very considerably.

HORACE G. DEMING.

UNIVERSTTY OF ILLINOIS.

\section{4,4'-DIMETHYL BENZOPHENONE AND ITS CONDENSATION WITH PHENOL.}

BY M. Gomberg and J. D. TOdd.

Received July 20, 1917.

4,4'-Dimethyl Benzophenone.-Carbon tetrachloride and benzene, under the influence of aluminum chloride, combine so as to give rise either wholly to triphenylchloromethane, ${ }^{1}$ or to benzophenone chloride, ${ }^{2}$ according as to whether benzene or carbon tetrachloride is in excess. The condensation of carbon tetrachloride with toluene is naturally more complex than with benzene, as various isomeric products can form simultaneously. None the less, with excess of toluene, fair yields (20 to $25 \%$ ) of tri-ptolyl chloromethane have been obtained, ${ }^{3}$ while with carbon tetrachloride largely in excess there results a mixture of isomeric tolophenone chlorides. ${ }^{4}$ On removing the aluminium chloride and evaporating the carbon tetrachloride, Boeseken obtained an almost colorless liquid which could not be distilled without partial decomposition. But on hydrolyzing the mixture of the various isomeric ketochlorides with acetic acid he succeeded in isolating pure $4,4^{\prime}$-dimethyl ketone.

We attempted to prepare 4,4'-dimethyl benzophenone chloride following the procedure of Gomberg and Jickling ${ }^{5}$ for benzophenone chloride, but found that the chloride suffered violent decomposition on distillation. Two mols toluene, mixed with an equal volume carbon tetrachloride, were allowed to drop into a suspension of aluminium chloride in about $300 \mathrm{cc}$. of carbon tetrachloride, the mixture being kept cold. After standing overnight, the aluminium chloride was decomposed with ice and, after drying over calcium chloride, the carbon tetrachloride was distilled off. On attempting to distil the ketochlorides in a vacuum, violent decomposition took place at about $175^{\circ}$, liberating large quantities of hydrochloric acid and forming a green resinous mass. The ketochlorides, without

I This JourNal, 22, 752 (1900).

2 Boeseken, Rev. trav. chim., 24, I (I905).

3 Gomberg and Voedisch, This Journal, 23, 177 (Igor).

4 Boeseken, Loc. cit., p. 4.

5 This Journal, 37, 2577 (I915). 
being subjected to vacuum distillation, were therefore hydrolyzed to the corresponding ketones by steam distillation for one hour. The solid residue was taken up in carbon tetrachloride, dried over calcium chloride and, after evaporating the solvent, distilled, coming over at $175-180^{\circ}$, under a pressure of $7-9 \mathrm{~mm}$. About two-thirds of the product can be distilled, the remainder decomposing. The distillate consists of about equal quantities of solid 4,4'-dimethyl benzophenone and a liquid which is probably a mixture of several isomeric tolophenones. An almost complete separation is effected by the addition of 20 to $30 \mathrm{cc}$. petroleum ether to the distillate. After standing several hours the crystals of $4,4^{\prime}$-dimethyl benzophenone are filtered off, washed with a little petroleum ether, and recrystallized from alcohol or petroleum ether to remove all traces of the liquid isomers. The ketone crystallizes as pure white needles melting at $93^{\circ}$. The yield is $30-35 \%$. The liquid portion distills at $320-322^{\circ}$ (uncorr.) $730 \mathrm{~mm}$. and is light yellow in color. The attempts to identify more completely the liquid ketones proved unsuccessful.

4,4'-Dimethyl Benzophenone Chloride.-4,4'-Dimethyl benzophenone and the calculated quantity of phosphorus pentachloride were heated two hours at $105^{-120^{\circ}}$. The phosphorus oxychloride was taken off under reduced pressure. On attempting to distil the ketochloride in a vacuum it decomposed above $175^{\circ}$, evolving hydrochloric acid and forming a green resinous mass, insoluble in alcohol or ether and only slightly soluble in benzene. Another sample of the ketone was treated in the same way and the oxychloride was removed under reduced pressure at II $5^{-120^{\circ}}$, leaving the ketochloride as a rather thick green liquid. Best results were obtained when the temperature was not allowed to rise at any time above $100^{\circ}$. Under these conditions the ketochloride was obtained as a thin green liquid.

Boeseken ascribed the decomposition, which occurs when one attempts to distil the mixed ketochlorides, to the presence in the mixture of some ortho-tolyl ketochloride. Evidently this explanation cannot be correct, as a decomposition of the same nature and at the same temperature takes place even with the pure di-para-tolophenone chloride.

Condensation with Phenol.-- $p$-Hydroxytriphenylcarbinol and a series of $o$-substituted $p$-hydroxytriphenylcarbinols have been reported as existing in two desmotropic forms. ${ }^{1}$ To determine the influence toward tautomerization of substitution in other benzene rings than that containing the hydroxyl group, the study of $4,4^{\prime}$-dimethyl-p-hydroxytriphenylcarbinol was undertaken. The condensation of the dimethyl benzophenone chloride with phenol was found to proceed in stages, as has been shown by Gomberg and Jickling to be the case with benzophenone chloride

${ }^{1}$ Gomberg, This Journal, 35, ro35 (I9I3); Gomberg and Van Stone, Ibid., 38, $1577(1916)$. 
itself. According to the conditions of the experiment either diphenoxyditolylmethane or ditolyl-p-hydroxyphenylcarbinol may be obtained.

Diphenoxy-ditolylmethane, $\left(\mathrm{CH}_{3} \cdot \mathrm{C}_{6} \mathrm{H}_{4}\right)_{2} \mathrm{C}\left(\mathrm{OC}_{6} \mathrm{H}_{5}\right)_{2}$. - To $6.7 \mathrm{~g}$. (I mol) of $4,4^{\prime}$-dimethyl benzophenone chloride in $30 \mathrm{cc}$. of dry benzene were added $5.5 \mathrm{~g} .\left(2^{1 / 2} \mathrm{mols}\right)$ of phenol in an equal volume of benzene. The reaction was allowed to proceed at room temperature, driving out the hydrochloric acid gas formed by a slow current of dry air. At the end of twelve hours ammonia was added to neutralize any hydrochloric acid remaining, the reaction mixture steam distilled to remove excess of phenol, and the residue was digested with $4 \%$ sodium hydroxide solution in order to remove any hydroxycarbinol that might have been present. On the addition of a few cc. of alcohol the mass often crystallizes. The crude diphenoxy compound is best crystallized from alcohol containing a few drops of ammonia, giving small white crystals melting at $\mathrm{r}_{32-\mathrm{r}} 33^{\circ}$. The yield is $70-75 \%$. It is only slightly soluble in alcohol, soluble in petroleum ether, and very soluble in ether, carbon tetrachloride, benzene, toluene, and acetone.

The compound is very sensitive to acids. A few minutes' warming on the steam bath with dilute hydrochloric acid hydrolyzes it completely into $4,4^{\prime}$-dimethyl benzophenone and phenol. The latter was identified by conversion into picric acid.

4,4'-Dimethyl-p-hydroxytriphenolcarbinol, $\left(\mathrm{CH}_{3} . \mathrm{C}_{6} \mathrm{H}_{4}\right)_{2} \mathrm{C}(\mathrm{OH}) \mathrm{C}_{6} \mathrm{H}_{4} . \mathrm{OH}$. $-6.7 \mathrm{~g}$. (I mol) of $4,4^{\prime}$-dimethyl benzophenone chloride and $5.5 \mathrm{~g} \cdot\left(2^{1} / 2\right.$ mols) of phenol were placed in a round-bottom flask, protected from moisture by a calcium chloride tube, and kept at a temperature of $50-60^{\circ}$. At the end of twelve hours sufficient ammonia was added to neutralize the hydrochloric acid and the reaction mixture steam distilled to remove excess of phenol. The carbinol remained as a light yellow oil which solidified on cooling. It was digested with warm $4 \%$ sodium hydroxide solution, cooled and filtered. Any ketone remaining in solution was taken out by shaking the filtrate with a small amount of ether. After removing the ether by the passage of a rapid stream of air, the carbinol was precipitated by carbon dioxide. On standing the flocculent precipitate becomes more granular and can be filtered. The yield is $85-90 \%$.

Unexpected difficulties were encountered in the further purification of this carbinol. All possible solvents, as well as mixtures of these, were tried. In all of these the carbinol dissolves readily, coming out invariably as an oil which would not solidify, even on long standing. Even the acetyl and benzoyl derivatives could not be obtained other than amorphous. Under these circumstances the methods which have been employed successfully for the isolation of the two desmotropic forms in the case of other carbinols could not avail in this instance, for this carbinol would not crystallize either from alcohol or from acetic acid. 
We believe that the two forms, each amorphous, were obtained, however, by the following methods: To obtain the benzenoid modification, the carbinol was redissolved in twice the theoretical quantity of $4 \%$ sodium hydroxide solution, filtered and diluted to several times its volume by water (Under some conditions of concentration the sodium salt of the carbinol is likely to separate and can only be gotten into solution again by long heating.) The carbinol was precipitated from its solution by carbon dioxide, allowed to stand overnight, filtered and washed with a large volume of water. After air drying for 48 hours on a porous plate in an atmosphere free from acid, it was placed in a vacuum desiccator containing a dish of soda lime and allowed to come to a constant weight. The carbinol was a pale yellow, very fine powder.

The quinonoid modification was obtained by dissolving the carbinol in acetic acid containing a little water and pouring slowly into a large volume of cold water with rapid stirring. The precipitated carbinol was filtered, washed with a little water, and dried to a constant weight in the same manner as the benzenoid form. The carbinol was an orange-red powder, in contrast to the pale yellow color of the benzenoid form.

Calc. for $\mathrm{C}_{21} \mathrm{H}_{20} \mathrm{O}_{2}$ : C, $82.89 \% ; \mathrm{H}, 6.58 \%$. Found: Benzoid: C, $83.01 \%$; H, $6.8 \mathrm{I} \%$. Quinonoid: C, $83.1 \mathrm{r} \%$; $\mathrm{H}, 6.63 \%$.

On heating in a melting-point tube the benzenoid form turned red and fused together at about $70^{\circ}$ and gave off water at $125^{\circ}$, while the quinonoid form became dark red and fused together at about $65^{\circ}$ and evolved water rapidly above $90^{\circ}$.

Dehydration of the Two Forms of Carbinol.-The procedure and apparatus employed for the purpose have been described by Gomberg and Van Stone. One gram of each was placed in a porcelain boat and the temperature maintained at $58^{\circ}$ at first, and later raised to $95^{\circ}$ as the rate of loss of water slakened, because of the partial fusion of the compounds.

\begin{tabular}{cccc} 
Time, & \multicolumn{2}{c}{ Loss. } & \\
\cline { 2 - 3 } I hour & $0.48 \%$ & I.19\% & Temper. \\
3 hours & 0.87 & 1.65 & $58^{\circ}$ \\
6 hours & I.18 & I.75 & 58 \\
I2 hours & I.39 & 2.11 & 58 \\
24 hours & I.75 & 2.41 & 58 \\
48 hours & 2.32 & 2.45 & 58
\end{tabular}

The loss in water in the benzenoid form gradually became equal to that of the quinonoid form and after about fifteen days, each had lost about $90 \%$ of theory. The results thus indicate that the carbinol exists in the two tautomeric modifications

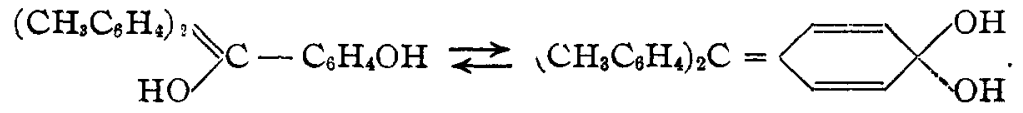




\section{Summary.}

I. Toluene and carbon tetrachloride, the latter in excess, condense, giving a mixture of isomeric tolophenone chlorides. On hydrolyzing the reaction mixture $4,4^{\prime}$-dimethylbenzophenone is obtained in yields of $35 \%$ of the calculated.

2. The di-p-tolophenone chloride, similarly to benzophenone chloride, combines with phenol without the aid of catalyst. The resulting $p$-hydroxytriarylcarbinol could only be obtained amorphous. However, enough evidence has been obtained to show that the carbinol exists in two tautomeric modifications, benzenoid and quinonoid.

ANN Arbor, Mich,

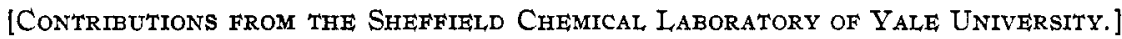

\section{RESEARCHES ON PYRIMIDINES. LXXXVI. THE PRODUCTION OF GLYOXALONES BY HYDROLYSIS OF PYRIMIDINE NUCLEOSIDES. ${ }^{1}$ \\ BY TREAT B. JoEnSON. \\ Received July 18, 1917.}

In recent papers from this laboratory ${ }^{2}$ it has been shown that certain types of hydroxypyrimidine compounds (secondary pyrimidine-nucleosides), which we have succeeded in synthesizing, can be transformed by hydrolysis with acids into derivatives of glyoxaline. A carbon atom is detached from the pyrimidine cycle in these compounds by hydrolysis with production of a five-membered ring in. which the urea group of the original pyrimidine remains intact. The nitrogen atoms of the urea nucleus couple with two carbon atoms in this change, to form a ring of which only one carbon functionated in the original pyrimidine cycle. A transformation of this type is illustrated by the following formulas expressing the rearrangement of the simplest secondary nucleoside of uracil (I) into dimethylglyoxalone ${ }^{3}$ (II):<smiles></smiles>

(I).

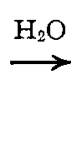<smiles>Cc1c[nH]c(=O)[nH]1</smiles>

(II).

${ }^{1}$ Since this paper was sent to the press an interesting paper has been published by Venable and Moore (Thrs JourNaL, 39, 1750) dealing with the oxidation of uric acid by hydrogen peroxide. They bring forward new data indicating that Scholtz did not have in hand tetracarbonimide but was actually dealing with cyanuric acid. Whether the tetracarbonimide is a precursor of this product remains to be established.

${ }^{2}$ Johnson and Hadley, This Journal, 38, 1844 (rgr6); Proc. Nat. Acad. Sci., 3, 418 (1917); ThIs JOURNAL, 39, 1715, 1919 (1917).

Johnson and Hadley, Loc. cit.; Kunne, Ber., 28, 2040 (I895); Biltz, Ibid., 40, $4801(1907)$. 